\title{
Measurement of High-Order Polarization Mode Dispersion
}

\author{
Yi Li, A. Eyal, P.-O. Hedekvist, and A. Yariv
}

\begin{abstract}
We demonstrate a new method to measure high-order polarization mode dispersion (PMD) using the Jones matrix exponential expansion. High-order PMD is characterized by measuring a series of characteristic matrices, which are convenient quantities for analyzing PMD effects in the time-domain. An experimental method is developed to estimate the validity range of the exponential expansion.
\end{abstract}

Index Terms-Differential group delay, optical fiber communication, optical measurements, polarization mode dispersion, principal states of polarization.

\section{INTRODUCTION}

$\mathbf{W}$ ITH the current trend toward ultrahigh bit rate transmission systems, the effects of second- and higher order PMD are becoming increasingly significant [1], [2]. It is thus important to find convenient ways to characterize these effects and measure them. Among the existing standard techniques are the Jones matrix eigenanalysis (JME) [3] and the Poincaré sphere technique (PST) [4], [5], and they are well-suited for accurate measurement of first-order PMD. Main drawbacks of these approaches include practical complexity to accommodate high-order PMD measurement and inconvenience for time-domain analysis such as calculating pulse deformation. Recently, Jopson et al. proposed a new measurement method, the Müller matrix method (MMM) [6] to measure higher order PMD. It attains algorithmic simplicity by staying completely in the Stokes space and is also capable of obtaining relatively high signal-to-noise ratio by requiring less contingent control of the input polarization states. However, the inconvenience for time-domain analysis still remains. In a recent paper [7] we have introduced an alternative method for characterizing high-order PMD, which is based on a novel expansion of the Jones matrix. The main advantage of this method is that the descriptors used to charaterize high-order PMD are directly related to PMD-induced pulse deformation. Therefore, they are particularly convenient for time-domain analysis. Here we describe a method to measure these high-order PMD descriptors. Since our method only involves measuring the Jones matrix within the transmission bandwidth, it is easy to implement by using a commercially available polarization analyzer. An additional reward of our measurement approach is that it can give, in principle, all orders of PMD descriptors simultaneously. In this

Manuscript received October 25, 1999; revised February 14, 2000. This work was supported by the Office of Naval Research (Yoon Soo Park), the Air Force Office of Scientific Research (H. Schlossberg), and the Swedish-Foundation for International Cooperation in Research and Higher Education (STINT).

The authors are with the Department of Applied Physics, California Institute of Technology, Pasadena, CA 91125 USA.

Publisher Item Identifier S 1041-1135(00)05589-0. letter, we report measurements of high-order PMD descriptors up to the fourth order, and the issue of measurement accuracy is addressed. Finally we demonstrate a method to estimate the validity range of the Jones matrix expansion mentioned above. It is then found that by including more high-order PMD descriptors, we are able to give precise description of PMD over a wider frequency bandwidth.

\section{THE EXPONENTIAL EXPANSION}

Let $\vec{E}_{\text {in }}=\vec{A} e^{i \omega t}$ be the input optical field vector which is characterized by its transverse $x$ - and $y$-components, and $\vec{E}_{\text {out }}$ be the output field after traveling through a linear medium. The Jones matrix $\mathbf{T}(\omega)$ of the medium is then defined by: $\vec{E}_{\text {out }}=$ $\mathbf{T}(\omega) \vec{E}_{\text {in }}$. The exponential expansion of the Jones matrix around some carrier frequency $\omega_{0}$ takes the form [7]

$$
\mathbf{T}\left(\omega_{0}+\Omega\right)=\mathbf{T}_{0} e^{\Omega \mathbf{N}_{1}} e^{\frac{1}{2} \Omega^{2} \mathbf{N}_{2}} e^{\frac{1}{! !} \Omega^{3} \mathbf{N}_{3}} \ldots
$$

where $\mathbf{T}_{0}$ is the Jones matrix of the medium at $\omega_{0}$. The $2 \times 2$ complex matrices, $\mathbf{N}_{k}$ 's, are defined as the $k$ th-order characteristic matrix of PMD. We denote the (column) eigenvectors and eigenvalues of $\mathbf{N}_{k}$ as $\hat{\epsilon}_{k}^{( \pm)}$and $\alpha_{k}^{( \pm)}$, then (1) can be written in the canonical form: [7]

$$
\mathbf{T}\left(\omega_{0}+\Omega\right)=\mathbf{T}_{0}\left[\mathbf{P}_{1} \mathbf{Q}_{1} \mathbf{P}_{1}^{-1}\right]\left[\mathbf{P}_{2} \mathbf{Q}_{2} \mathbf{P}_{2}^{-1}\right] \ldots
$$

where

$$
\begin{aligned}
\mathbf{P}_{k} & \equiv\left[\begin{array}{ll}
\hat{\epsilon}_{k}^{(+)} & \hat{\epsilon}_{k}^{(-)}
\end{array}\right] \\
\mathbf{Q}_{k} & \equiv\left[\begin{array}{cc}
e^{\frac{1}{k !} \Omega^{k} \alpha_{k}^{(+)}} & 0 \\
0 & e^{\frac{1}{k !} \Omega^{k} \alpha_{k}^{(-)}}
\end{array}\right] .
\end{aligned}
$$

In a similar fashion to defining the PSP's and DGD to characterize the first-order PMD, we define $\hat{\epsilon}_{k}^{( \pm)}$as the $k$ th-order principal states of PMD, and $\operatorname{Im}\left\{\alpha_{k}^{(+)}-\alpha_{k}^{(-)}\right\}$as the kth-order Differential Group Delay Dispersion (DGDD) [7]. In the special case of $k=1, \epsilon_{1}^{( \pm)}$are the well-known PSP's, and the first-order DGDD is the ordinary DGD of the medium. The effect of second-order PMD is described by $\epsilon_{2}^{( \pm)}$ and $\mathrm{DGDD}_{2} \equiv \operatorname{Im}\left\{\alpha_{2}^{(+)}-\alpha_{2}^{(-)}\right\}$. The physical meaning of these descriptors can be best visualized in a medium in which the first-order PMD has been compensated. In such a medium the dominant effect of PMD is a quadratic phase difference between the second-order PSP's. This quadratic phase difference, given by $(1 / 2) \Omega^{2}(\mathrm{DGDD})_{2}$, is manifested in the time domain by a differential broadening of pulses. Pulses which are aligned with $\epsilon_{2}^{(+)}$and $\epsilon_{2}^{(-)}$will experience maximum and minimum broadening, respectively. The particular form of (2) makes it a convenient formulation for studying pulse deformation due 
to high-order PMD, since each bracket can be treated as a subsystem of different phase dispersion characteristics.

\section{Measurement Method}

Measuring the characteristic matrices is, therefore, important for characterizing high-order PMD. Here we describe an accurate and easy-to-implement method to measure these matrices. By explicitly expanding each exponential term in (1) and comparing the resulting expansion to the ordinary Taylor expansion of the Jones matrices

$$
\mathbf{T}\left(\omega_{0}+\Omega\right)=\mathbf{T}_{0}+\left.\mathbf{T}^{\prime}\right|_{\omega_{0}} \Omega+\left.\frac{1}{2} \mathbf{T}^{\prime \prime}\right|_{\omega_{0}} \Omega^{2}+\cdots
$$

we obtain $\mathbf{N}_{k}$ 's in terms of $\mathbf{T}$ and its derivatives

$$
\begin{aligned}
& \mathbf{N}_{1}=\left.\mathbf{T}_{0}^{-1} \mathbf{T}^{\prime}\right|_{\omega_{0}} \\
& \mathbf{N}_{2}=\left.\mathbf{T}_{0}^{-1} \mathbf{T}^{\prime \prime}\right|_{\omega_{0}}-\left(\mathbf{N}_{1}\right)^{2} \\
& \mathbf{N}_{3}=\left.\mathbf{T}_{0}^{-1} \mathbf{T}^{\prime \prime \prime}\right|_{\omega_{0}}-\left(\mathbf{N}_{1}\right)^{3}-3 \mathbf{N}_{1} \mathbf{N}_{2} .
\end{aligned}
$$

It is evident that measuring the characteristic matrices is equivalent to measuring the derivatives of the Jones matrix. In most polarization analyzing instruments the first-order derivative of $\mathbf{T}$, which is used subsequently to calculate the DGD, is obtained by measuring the difference of the Jones matrix at two nearby frequencies. In principle, high-order derivatives of the Jones matrix could be obtained similarly. In reality, however, this direct approach leads to significant errors in high-order derivatives since we are dealing with ratios of extremely small quatities, and minor measurement fluctuations may result in large deviation. Better accuracy can be achieved by numerically fitting the Jones matrix data to a polynomial of matrices $\mathbf{T}_{n}(\Omega)=\mathbf{A}_{0}+\mathbf{A}_{1} \Omega+\mathbf{A}_{2} \Omega^{2}+\cdots+\mathbf{A}_{n} \Omega^{n}$, the degree of which depends on the number of terms to keep in the expansion (1). The derivatives of the Jones matrix are then given by the coefficients of this polynomial: $\left.\mathbf{T}^{(k)}\right|_{\omega_{0}}=k ! \cdot \mathbf{A}_{k}$. In summary, the measurement comprises of the following stages: 1) The Jones matrix is measured at consecutive wavelengths in the bandwidth of interest; 2) A polynomial fit is performed to obtain an approximated analytical expression for the measured Jones matrix; 3) From the resulting fitting coefficients the characteristic matrices $\mathbf{N}_{k}$ 's are calculated.

Besides the simultaneity of determination of all orders of PMD descriptors, our approach allows for convenient verification of measurement accuracy by comparing the measured Jones matrix with the following truncated exponential expansion

$$
\mathbf{T}\left(\omega_{0}+\Omega\right)=\mathbf{T}_{0} e^{\Omega \mathbf{N}_{1}} e^{\frac{1}{2} \Omega^{2} \mathbf{N}_{2}} \ldots e^{\frac{1}{n !} \Omega^{n} \mathbf{N}_{n}} .
$$

If better agreement is achieved by including more exponential terms in (5), the accuracy of measured $\mathbf{N}_{k}$ 's is confirmed.

\section{EXPERIMENTAL RESULTS}

In the experimental setup, a tunable laser source was directed through a polarization analyzer and our system under test (SUT). The Jones matrix of the SUT was measured as function
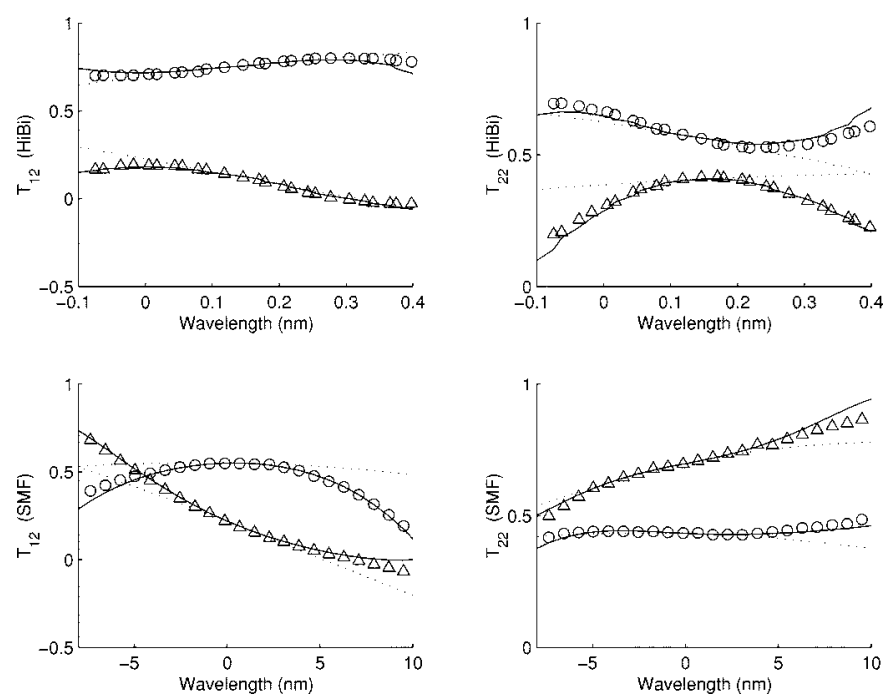

Fig. 1. Comparison of measured Jones matrix elements $T_{12}$ and $T_{22}$ (circles - real part; triangles - imaginary part) and reconstructed finite product (5). The dotted lines are for $n=1$ and the solid lines are for $n=3$. The wavelength is with respect to $\lambda_{0}=1546.5 \mathrm{~nm}$.

of wavelength in a suitable bandwidth. The measurement was performed on two different types of fibers. One is a standard 33 $\mathrm{km}$ single-mode fiber (SMF). The other one is made of three concatenations of high-birefringence fibers (HiBi). Both are polarization-mode dispersive media, and the average PMD is a few pico-second for the HiBi and a few tenth pico-second for the SMF. The inclusion of the HiBi fiber enables us to compare our measurement to known analytic results later.

For each SUT the characteristic matrices were calculated from the measured Jones matrix, and truncated products (5) for different $n$ values are constructed and compared against the original Jones matrix data (Fig. 1). For both HiBi and SMF, the truncated product approximation improves with the inclusion of more terms in (5). The major improvement in agreement between the Jones matrix and its exponential expansion that results from the inclusion of the $n=3$ term strongly confirms the validity of the exponential expansion in describing pulse propagation. Since the propagator (5) is in the form of products of operators, it lends itself more readily to optical manipulation and compensation by a serial arrangement of optical components.

Further confirmation of accuracy can be achieved by comparing the DGD profiles calculated from the truncated products (5) and that obtained from the measurement, as shown in Figs. 2 and 3. Once again, the inclusion of more terms in the truncated product approximation leads to better agreement with the measurement.

To assess the relative importance of different orders of PMD effects, it is instrumental to estimate the validity range (VR) of the truncated product approximation for a given $n$. The definition of the VR is somewhat arbitrary, and here we define that its boundary is where the DGD calculated from (5) starts to deviate rapidly from a reference DGD curve. In real applications, this reference is just the DGD data from the measurement. Figs. 2 and 3 illustrate the applicability of this approach, and it is evident that inclusion of more higher order 


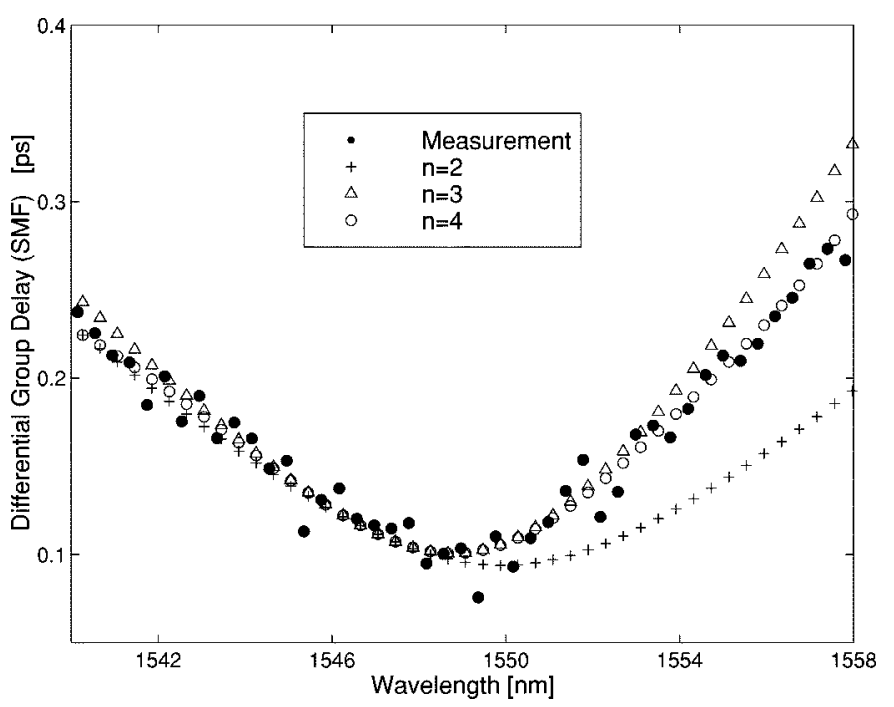

Fig. 2. Comparison between the measured DGD profile and those calculated from the truncated products (5) for the SMF.

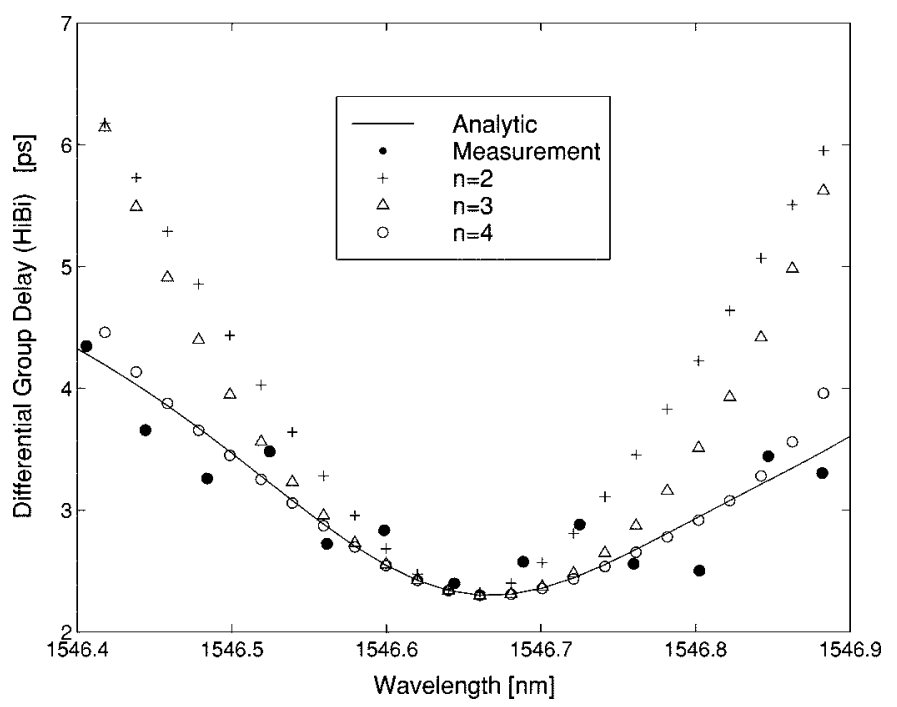

Fig. 3. Comparison between the measured (and analytical) DGD profile and those calculated from the truncated products (5) for the HiBi.

terms in (5) leads to wider VR's. The VR's for different $n$ values are listed below

$$
\begin{array}{cccc}
n & 2 & 3 & 4 \\
\text { VR in nm (HiBi): } & 0.05 & 0.15 & 0.35 \\
\text { VR in nm (SMF): } & 4.0 & 8.0 & 15.0 .
\end{array}
$$

Finally, it is interesting to compare the results from measurement and the analytical predictions for the concatenations of HiBi fibers. By using the analytical formula derived by Gisin and Pellaux [8], we can express the DGD of concatenations of birefringent fibers as a function of fiber parameters such as coupling angles and individual DGD's of each concatenation. Due to extreme sensitivity of the DGD profile on these parameters, the exact values of these parameters are fine-tuned around their experimentally measured values by numerically fitting the analytical DGD curve to measurement data. The solid curve in Fig. 3 is the analytical DGD profile with numerically determined coupling angles of $45.02^{\circ}$ and $-45.20^{\circ}$, and individual DGD's of $6.1314 \mathrm{ps}, 2.3690 \mathrm{ps}$, and $6.1312 \mathrm{ps}$. The analytical curve fits well with the measurement results.

\section{CONCLUSION}

The exponential expansion of the Jones matrix is a new technique that provides a convenient and intuitive way to analyze high-order PMD effects. By using a small number of descriptors (either the characteristic matrices or the corresponding PSP's and DGDD's) the PMD properties of the transmission medium can be completely described within a given range of frequencies. We have demonstrated an accurate method to measure the PMD characteristic matrices. By showing the convergence of the finite product approximation (5) to the experimental measurement, we have not only verified the applicability of the new Jones matrix exponential expansion experimentally, but also confirmed the accuracy of our method. The validity range of the finite product approximation has been estimated as a guideline for applying the measured descriptors to time-domain analysis.

\section{ACKNOWLEDGMENT}

The authors would like to thank K. Vahala for kindly providing the polarization analyzer.

\section{REFERENCES}

[1] C. D. Poole and T. E. Darcie, "Distortion related to polarization mode dispersion in analog lightwave systems," J. Lightwave Technol., vol. 11, pp. 1749-1759, Nov. 1993.

[2] P. Ciprut, B. Gisin, N. Gisin, R. Passy, J. P. Von der Weid, F. Prieto, and C. W. Zimmer, "Second-order polarization mode dispersion: Impact on analog and digital transmissions," J. Lightwave Technol., vol. 16, pp. 757-771, May 1998.

[3] B. L. Heffner, "Accurate, automated measurement of differential group delay dispersion and principal state variation using jones matrix eigenanalysis," IEEE Photon. Technol. Lett., vol. 5, pp. 814-817, July 1993.

[4] D. Andresciani, F. Curti, F. Matera, and B. Daino, "Measurement of the group-delay difference between the principal states of polarization on a low-birefringence terrestrial fiber cable," Opt. Lett., vol. 12, pp. 844-846, Oct. 1987.

[5] C. D. Poole, N. S. Bergano, R. E. Wagner, and H. J. Schulte, "Polarization dispersion and principal states in a $147 \mathrm{~km}$ undersea lightwave cable," J. Lightwave Technol., vol. 6, pp. 1185-1190, July 1988.

[6] R. M. Jopson, L. E. Nelson, and H. Kogelnik, "Measurement of second-order polarization-mode dispersion vectors in optical fibers," IEEE Photon. Technol. Lett., vol. 11, pp. 1153-1155, Sept. 1999.

[7] A. Eyal, W. K. Marshall, M. Tur, and A. Yariv, "A new representation of second order polarization mode dispersion," Electron. Lett., vol. 35, pp. 1658-1659, Oct. 1999.

[8] N. Gisin and J. P. Pellaux, "Polarization mode dispersion: Time versis frequency domains," Opt. Commun., vol. 89, pp. 316-323, 1992. 